\title{
A Directional Antenna Medium Access Control Protocol for Wireless Ad Hoc Networks
}

\author{
Carlos de M. Cordeiro, Hrishikesh Gossain, and Dharma P. Agrawal
}

\begin{abstract}
-
In this paper we propose a Directional Antenna Medium Access (DAMA) protocol that takes advantage of the benefits offered by directional antennas and is best suited for sparsely distributed networks. A large portion of DAMA has been inspired by the IEEE 802.11 MAC, with major enhancements including a new neighbor discovery scheme, and an optimized circular directional transmission of RTS and CTS to prevent the hidden node problem, reduce collisions and decrease node deafness. In addition, a pair of communicating nodes using DAMA simultaneously transmits the circular directional RTS and CTS only to those sectors with neighbors, hence reducing overall communication delay and enhancing throughput. We have compared DAMA with IEEE 802.11 and two recently proposed directional MAC protocols, and results show that DAMA performs better than these protocols in the majority of the scenarios investigated while we point out that the performance depends on the network topology and traffic pattern.
\end{abstract}

Keywords: 802.11, MAC, Directional Antenna, Spatial Reuse, Wireless Ad Hoc Networks.

\section{INTRODUCTION}

Most of the existing research on ad hoc networks typically assumes the use of omni directional antennas by all nodes. Such an example is the IEEE 802.11 medium access control (MAC) [1] protocol which appears to efficiently solve the issues of this type of environment. However, due to the omni directional nature of transmissions, network capacity is considerably limited. For example, the distribution of energy in all directions other than the intended direction not only generates unnecessary interference to other nodes, but also decreases the potential range of transmissions. With directional communications, on the other hand, both range and spatial reuse can be substantially enhanced, by having nodes concentrate transmitted energy only towards their destination's direction. On the receiving side, directional antennas enable a node to selectively receive signals only from the certain desired direction, thereby increasing the signal to interference and noise ratio (SINR).

Carlos de M. Cordeiro is with Nokia Research Center. Hrishikesh Gossain is with the Center for Distributed and Mobile Computing of the University of Cincinnati. Dharma P. Agrawal is with the Department of ECECS of the University of Cincinnati. E-mails: carlos.cordeiro@nokia.com, dpa@ececs.uc.edu.
Therefore, traditional MAC protocols which have been designed under the omni directional assumption [1, 2] are no longer suitable for use over directional antennas. The design of an efficient MAC protocol for directional antennas is then a crucial issue and needs further investigation. In directional antennas, new types of hidden node problems arise [3]. In addition, issues such as node deafness and the determination of neighbors' locations have to be properly handled [4]. A detailed study that analyzes several important aspects regarding directional antennas and the factors that affect them can be found in [13].

In this paper, we introduce a directional antennas medium access (DAMA) control protocol for use over directional antennas. DAMA addresses the hidden node problem and node deafness by employing a novel scheme of selective circular directional transmission of RTS and CTS, where these packets are transmitted only through the antennas with neighbors. For that, DAMA employs a selflearning algorithm to determine the presence or absence of nodes in given directions. Moreover, we point out the deficiencies in existing MAC protocols proposed for directional antennas and show how DAMA overcomes such problems. It is worthwhile to note that DAMA is best suitable for sparsely distributed networks. In a dense network, chances of having a neighbor in each antenna beam are high which may result into considerable RTSCTS sweeping overhead.

The rest of this paper is organized as follows. In Section 2 we discuss the related work on MAC protocols for directional antennas. The antenna model and a glimpse of IEEE 802.11 are then given in Section 3. In Section 4 we present two recent proposed MAC protocols for directional antennas and point out deficiencies in their design. Next, Section 5 thoroughly describes our proposed DAMA protocol and how it overcomes the problems discussed. Comprehensive simulation study and comparison of DAMA with three other MAC protocols including IEEE 802.11 is given in Section 6. Finally, this paper is concluded in Section 7 highlighting some open problems and future research plan.

\section{RELATED WORK}

Most of the research in the area of directional antennas has focused broadband and cellular networks [5, 6, 7]. In the context of wireless ad hoc networks, research is still at its infancy. In general for ad hoc networks, two models for MAC protocols for directional antennas can be identified. In the first model [8], each node is equipped with $M$ antennas whose orientations can be maintained at any time, regardless of the node's movement. In this model, it is assumed that nodes have directional reception capability, i.e., they can activate the antenna pointing to the direction of the desired destination while deactivating antennas in all 
other directions. Thus, the receiving node is not influenced by simultaneous transmissions from other nodes as long as it is not received at the antenna beam the receiver is currently listening to. Most recent research adopts this model [3, 4, 8]. In the second model [9], antennas are always active for receiving and thus transmissions to different antennas results in collision. Some MAC proposals for directional antennas assume this model [10]. In this work, we consider the first model and elaborate on the same in the next section. We also describe its shortcomings and how our proposed protocol overcomes them.

In [8], a variation of RTS/CTS mechanism of IEEE 802.11 adapted for use with directional antennas is given. This protocol sends the RTS and CTS packets omnidirectionally in order to enable the transmitter and receiver to locate each other, and sends the DATA and ACK packets in directional mode. A MAC protocol that sends a directional RTS and an omnidirectional CTS is presented in [11]. Here, it is assumed that the transmitter knows the receiver's location, so that it can send the RTS directionally. In case location information is not available, the RTS is transmitted in omni mode in order to find the receiver. In [12] it is proposed the use of Directional Virtual Carrier Sensing in which directional RTS and CTS transmissions are employed. Here, it is assumed that the transmitter knows the receiver's location. Similarly to [11], RTS are transmitted omni-directionally in case location information is not available. Finally, [3] studies the problems that appear using directional antennas and proposes a MAC protocol to take advantage of the higher gain obtained by directional antennas. This protocol employs a scheme of directional multihop RTS transmissions so as to establish directional-directional (DD) links between the transmitter and receiver. An assumption of this scheme is that the transmitter must know the entire route to the intended receiver so that the RTS packet can be routed.

The protocols aforementioned share common characteristics that lead to several inefficiencies. In $[8,11$, 13] at least one omnidirectional transmission of a control packet is employed, hence limiting the coverage area. The presence of omnidirectional transmissions of either RTS or CTS limit the range of directional transmissions, which is now defined by the smaller coverage range between any of these packets. This situation is shown in Figure 1 and constitutes a disadvantage of these schemes, as they do not exploit the increased coverage range provided by directional transmissions. In other words, given a particular transmit energy, an array of $M$ antenna beams provides an increased antenna gain in comparison with the omni mode of the order of $M[14,15,16]$. This gain is doubled if there is directivity in both transmission and reception. Thus, a directional communication between two stations may significantly increase the distance between them as compared to the equivalent omni communication, a benefit that has not been explored by the above schemes.

In addition, although $[3,12]$ uses directional transmissions only, they do not solve the issues of increased instances of hidden terminal problem, node deafness and the determination of neighbors' location. The first two problems are thoroughly studied in [3], although a solution is not provided. The third problem originates from the fact that a node has to know through which antenna it can communicate with the intended receiver before transmitting a directional RTS. In [3, 12], nodes' location is assumed to be known beforehand, while [11] assumes nodes' location can be determined with the assistance of an additional hardware such as GPS.

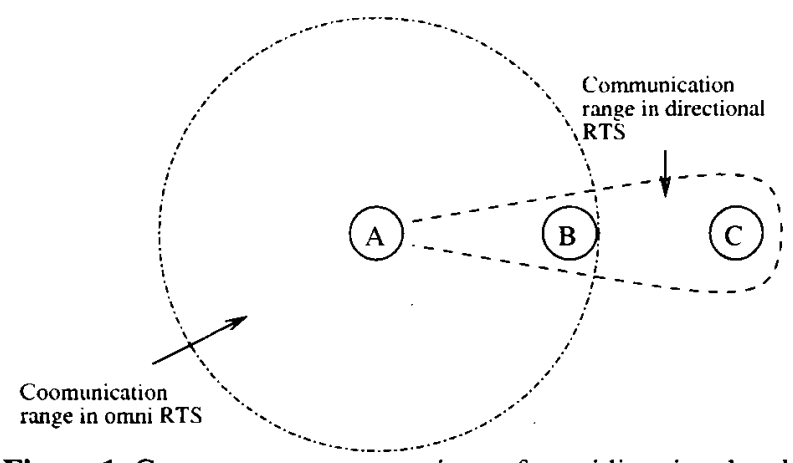

Figure 1. Coverage range comparison of omnidirectional and directional transmissions

In $[3,9]$ a protocol called Directional MAC (DMAC) is proposed that employs directional transmission of RTS and CTS. Similar to the previous schemes, it assumes nodes' locations are known a priori. This protocol also suffers from node deafness and hidden node problems [4].

To overcome the shortcomings in DMAC, it is proposed in [4] a scheme that employs directional transmission of RTS and CTS without previous neighbors' location knowledge. To accomplish that, a scheme of circular directional transmission of RTS is carried out by the transmitter which ensures that the RTS packet will eventually reach the intended destination. The destination then sends back a single directional CTS packet towards the source. We refer to this scheme as Circular RTS MAC (CRM). While CRM does not assume prior neighbor's location availability, it does not satisfactorily prevent node deafness and collisions. As we discuss later, CRM has shortcomings which may result in poor performance.

Finally, $[3,4,11,12]$ propose the concept of Directional Virtual Carrier Sense (DVCS) and Directional Network Allocation Vector (DNAV) mechanisms that is similar to the DNAV concept employed in our DAMA protocol and is discussed later.

\section{PRELIMINARIES}

\subsection{THE ANTENNA MODEL}

We have implemented a complete and flexible directional antenna module at the Network Simulator (NS version 2.26) [17]. This model possesses two separate modes: Omni and Directional. This may be seen as two separate antennas: an omni-directional and a steerable single beam antenna which can point towards any specified directions [3].In principle, both the Omni and Directional modes may be used to transmit or receive signals. However, in our proposed MDA protocol, the Omni mode is used only to receive signals, while the Directional mode is used for transmission as well as reception. This way, both 
transmitter and receiver take advantage of the increased coverage range provided by beamforming.

In Omni mode, a node is capable of receiving signals from all directions with a gain of $\mathrm{G}^{\mathrm{O}}$. While idle (i.e., neither transmitting nor receiving), a node stays in Omni mode when using our proposed protocol. As soon as a signal is sensed a node can detect the direction through which the signal is strongest and goes into the Directional mode in this particular direction.

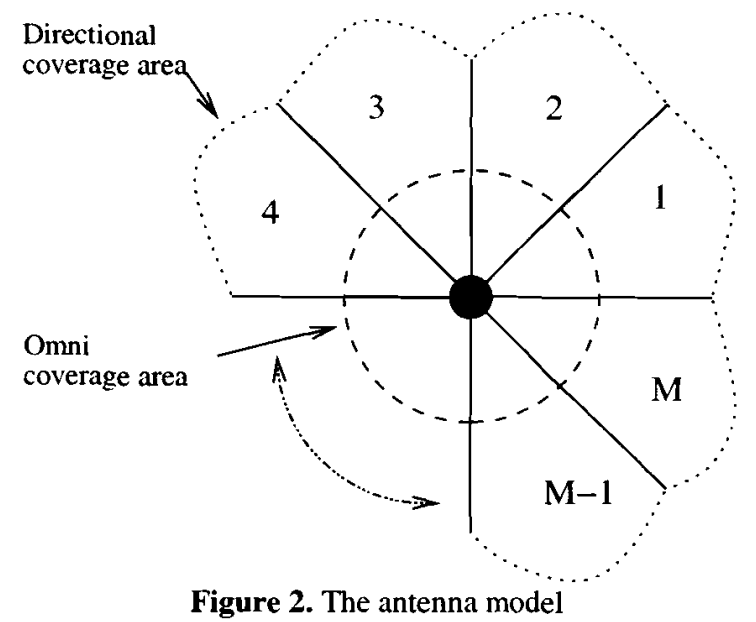

In Directional mode, a node can point its beam towards a specified direction with gain $G^{d}$ (with $G^{d}$ typically greater than $\mathrm{G}^{\mathrm{O}}$ ). In addition, the gain is proportional to number of antenna beams (i.e., inversely proportional to the beamwidth) given that more energy can be focused on a particular direction, thus resulting in increased coverage range. A Node provides coverage around it by a total of $\mathbf{M}$ non-overlapping beams (Figure 2). The beams are numbered from 1 through $M$, starting at the three o'clock position and running counter clockwise. In Directional mode, and at a given time, a node can transmit or receive in only one of these antenna beams. In order to perform a broadcast, a transmitter may need to carry out as many directional transmissions as there are antenna beams so as to cover the whole region around it. This is called sweeping. In the sweeping process, we assume there is only carrier sensing delay in beamforming in various directions. This model has been widely studied in the literature $[3,4,8$, 9].

To simplify modeling of antenna side lobes, we assume that energy contributed to the side lobes is uniformly distributed in a circular area. Although the amount of energy contributed to the side lobes depends on the type of antenna been used as well as number of beams been supported [13], in our simulation we assume that the side lobe gain is fixed and is set to -20dBi. Finally, we assume that all nodes use the same directional antenna patterns and can maintain the orientation of their beams at all times [8].

\subsection{THE IEEE 802.11}

In the IEEE 802.11 [11], the Distributed Coordination Function (DCF) coordinates medium access in ad hoc networks. In DCF, an RTS and CTS handshake precedes
DATA communication and the following ACK. DCF in IEEE 802.11 conducts two forms of carrier sensing: physical (by listening to the wireless shared medium) and virtual. Virtual carrier sensing uses the duration field which is included in the header of RTS and CTS frames. The duration included in each of these frames can be used to determine the time when the source node would receive an ACK frame from the destination node. This duration field is utilized to set a station's Network Allocation Vector (NAV), which indicates the remaining time the medium is busy with the ongoing transmission. Using the duration information, nodes update their NAVs whenever they receive a packet. The channel is considered to be busy if either physical or virtual carrier sensing (by the NAV) so indicates. Whenever NAV is zero, a station may transmit if the physical sensing allows.

The area covered by the transmission range of the sender and receiver is reserved for data transfer, and hence other nodes cannot initiate transmission while communication is in progress. Given this fact, this region is hereby referred to as silenced region. By using the RTS and CTS handshake to silence the nodes in the silenced region, IEEE 802.11 is able to overcome, although not completely [20], the hidden terminal problem [18, 19].

The IEEE 802.11 MAC protocol uses a backoff mechanism to resolve channel contention. Before initiating a transmission, if a node $\mathrm{S}$ senses the medium busy, it chooses a random backoff interval from $[0, \mathrm{CW}]$, where $\mathrm{CW}$ is called contention window. After every idle "slot time", node S decrements the backoff counter by one. When it reaches zero, node $S$ can transmit the packet. If collision occurs with some other transmission, $\mathrm{S}$ doubles its $\mathrm{CW}$, chooses a new backoff interval and tries retransmission. If during the backoff stage the medium is sensed busy, the node freezes its backoff and resumes it once the medium has become idle for a duration called DIFS (DCF interframe space). The backoff procedure is only invoked when the medium has been sensed idle for DIFS duration. A shorter interframe space (SIFS) is used to separate transmissions belonging to a single dialogue (i.e., to separate RTS-CTSDATA-ACK transmissions).

\section{THE DMAC AND CRM PROTOCOLS}

The IEEE 802.11 limits spatial reuse of the wireless channel by having nodes in the neighborhood of a sender and receiver pair to remain silent while communication is in progress. With directional antennas, however, it may be possible to conduct multiple simultaneous transmissions in the same neighborhood. For example, in Figure 3 node pairs $\mathrm{A}$ and $\mathrm{B}$, and $\mathrm{C}$ and $\mathrm{D}$ can communicate simultaneously provided the beamwidth of the directional transmissions is not very large. However, simultaneous communication between nodes $\mathrm{E}$ and $\mathrm{F}$, and nodes $\mathrm{A}$ and $\mathrm{B}$ is not possible.

As we have seen earlier, due to higher antenna gain, directional antennas have a greater transmission range than omnidirectional antennas. This enables distant nodes to communicate over a single hop, and results in increased throughput and reduced delay. 
A MAC protocol for directional antennas should attempt to take advantage of both benefits of directionality: spatial reuse and higher transmission range. The DMAC protocol described in the next section attempts to achieve both spatial reuse of the channel and take advantage of the higher transmission range by using directionalomnidirectional (DO) links. We then show that DMAC has its own problems and limitations, and then introduce CRM. The CRM protocol tries to overcome some of the limitations of DMAC. We show that CRM itself introduces another set of issues and does not completely tackle the deficiencies in DMAC.

\subsection{THE DMAC PROTOCOL}

The DMAC protocol assumes nodes know their neighbors' location, that is, they are aware through which antenna beam a given neighbor can be reached. Channel reservation in DMAC is performed using a RTS/CTS handshake, both being transmitted directionally. An idle node listens to the channel in Omni mode, i.e., omnidirectionally. Whenever a node receives a signal from a particular direction, it locks onto that signal directionally and receives it. Please note that collisions may happen during signal reception, while the node finds itself in Omni mode. Only when a node is beamformed in a specific direction, it can avoid interference in the other remaining directions.

(C)

(A)
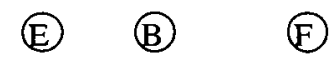

(D)

Figure 3. Directional communication example

The RTS transmission in DMAC is as follows. Before sending a packet, the transmitter node $S$ performs a directional physical carrier sensing towards its intended receiver $R$. If the channel is sensed idle, DMAC checks its Directional NAV (DNAV, explained later in this paper) table to find out whether it must defer transmitting in the direction of node $R$. The DNAV (elaborated later in this paper) maintains a virtual carrier sense for every Direction of Arrival (DoA) (i.e., for every antenna beam) in which it has overheard a RTS or CTS packet. If node S finds it is safe to transmit, it performs similar to IEEE 802.11 by entering the backoff phase and transmitting the packet in the direction of node $R$ when the backoff counter counts down to zero.

If idle, the receiver node $R$ remains in Omni mode listening to the channel omnidirectionally. When node $R$ receives the RTS from $S$, it is able to detect the DoA of the RTS and lock in the corresponding direction. Upon complete reception of the RTS packet, node $R$ beamforms in the direction of node $S$ and sends the CTS packet directionally towards $S$ provided its DNAV indicates it is free to do so. Similar to IEEE 802.11, the CTS is transmitted after SIFS duration after reception of the RTS.
Note that the nodes other than $S$ and $R$, say $X$, which receive either the RTS or CTS packet, , updates its DNAV in the captured DoA with the duration field specified in the RTS or CTS packet. This prevents node $X$ from transmitting any signal in the direction which may interfere with the ongoing transmission between nodes $S$ and $R$.

\subsubsection{PROBLEMS WITH DMAC}

As described in [3], two of the main problems with DMAC, which are also common in other directional MAC protocols, are:

Hidden terminal problems - In IEEE 802.11 RTS/CTS packets are transmitted omnidirectionally to overcome the hidden terminal problem, while this is not the case in DMAC. There are two main sources of hidden terminal problems, namely, hidden terminal problem due to asymmetry in gain and hidden terminal problem due to unheard RTS/CTS. The first problem is due to the fact that nodes which are in Omni mode, have a smaller gain as compared to nodes which are Directional mode. When nodes in Omni mode go into Directional mode (e.g., to transmit a RTS), they may be unaware of an ongoing transmission and a collision may take place. The second problem comes from the reverse situation. That is, a node in Directional mode cannot listen to any other transmission in a direction other than where it is beamformed. Therefore, when this node goes into Omni mode it may transmit towards a direction where a transmission is being carried out. Obviously, these problems do not occur in omnidirectional transmissions as all neighbor nodes potentially become aware of any nearby transmission. We can see that there is a clear tradeoff between spatial reuse and collisions when employing directional antennas.

Deafness - Lets assume that two nodes, say $S$ and $R$, are currently beamformed in each other direction, that is, they are in Directional mode. A third node $C$ which has not heard to the RTS/CTS from nodes $S$ and $R$ and which has a packet to send to either of them, will keep on transmitting RTS to its desired destination, say node $R$. Since node $R$ is beamformed in the direction of node $S$, it is deaf in the direction of node $C$ and does not respond to its RTS. Therefore, node $C$ will keep on transmitting the RTS towards node $R$ until the number of attempts exceeds a threshold name Short Retry Limit (SRL) (defined in IEEE 802.11), when it then reports the failure to the routing layer which takes the necessary actions. In addition, this may result in unfairness as the backoff interval is doubled upon every failed transmission. This problem is referred to as the deafness, since node $R$ is deaf to the signals from node $C$ while $R$ is beamformed in the direction of $\mathrm{S}$. The deafness problem results in excessive wastage of network capacity in unproductive transmissions, and in increased energy consumption.

\subsection{THE CRM PROTOCOL}

The CRM protocols attempts to overcome the limitations found in DMAC. Contrary to DMAC, CRM does not depend on the availability of neighbors location information. To accomplish that, CRM employs a circular directional transmission of the RTS packet, that is, a node $S$ 
with an RTS to be sent to node $R$ directionally transmits the same through all antenna beams. This way, node $R$ will eventually receive the RTS packet coming from node $S$.

Also based on this scheme, CRM may decrease the occurrence of node deafness as it informs all nodes within the transmitter's directional radio range about the oncoming transmission. This way, nodes overhearing the RTS defer their transmission in the direction of the transmitter, hence minimizing deafness. In addition, CRM includes extra information in the RTS and CTS packets so as to enable other nodes to determine whether they need to defer in the direction of the transmitter or receiver, thus also minimizing the hidden terminal problem.

Upon receipt of an RTS packet, the receiver node $R$ delays the transmission of its CTS for a period of $\mathrm{T}_{\mathrm{CRM}}=\mathrm{K}$ * RTS_Transmission_Time + SIFS, where $K$ is the number of antenna beams the sender node $S$ will transmit the circular directional RTS, RTS_Transmission_Time is the time required for the transmission of a single RTS, and SIFS is as described earlier. Therefore, the CTS is only transmitted when the sender node has swept through its entire antenna beams.

\subsubsection{PROBLEMS WITH CRM}

CRM protocol does not completely overcome the limitations of DMAC, and itself introduces new shortcomings. First of all, CRM only prevents node deafness in the neighborhood of the transmitter node. As we have seen earlier, CRM employs a circular directional RTS transmission and a single directional CTS transmission. As a result, CRM is only able to cope up with node deafness at the sender neighborhood, while deafness may still occur in the neighborhood of the receiver.

A more serious issue with CRM is in the design of its RTS/CTS handshake. In CRM, a sender node $S$ initiates the circular directional transmission of its RTS although it is not at all sure whether its intended receiver node $R$ has correctly received its RTS or not. Consider the example in Figure 4 where nodes are equipped with an eight-beam antenna array. Further consider that the sender node $S$ initiates transmission of a circular RTS through antenna one and its intended destination node $R$ is located at the antenna six. As node $S$ circularly transmits the RTS packets, nodes in the corresponding directions update their DNAV for the duration contained in the RTS packet. Now assume that when node $S$ transmits its RTS through antenna six towards node $R$, node $A$ also sends a RTS to node $\mathrm{R}$ thus causing a collision. In this case, node $R$ will not respond to node $S$ 's RTS. The side effect of this is that nodes in the neighborhood of node $S$ and which correctly receive the circular RTS will not be able to initiate any transmission either towards node $S$ or node $R$, since their DNAV is set towards both nodes $S$ 's and $R$. Clearly, this degrades the network capacity.

Another limitation in CRM can also be seen through the example in Figure 4. Here, we see that the sender node $S$ transmits its circular directional RTS through four "empty" sectors. That is, out of the eight sectors covered by the eight antenna beams of node $S$, four of them have no neighbors. Therefore, for every circular RTS transmission node $S$ wastes four of them. As shown in our simulation studies, this overhead has an increasingly larger impact as the number of antenna beams is increased.

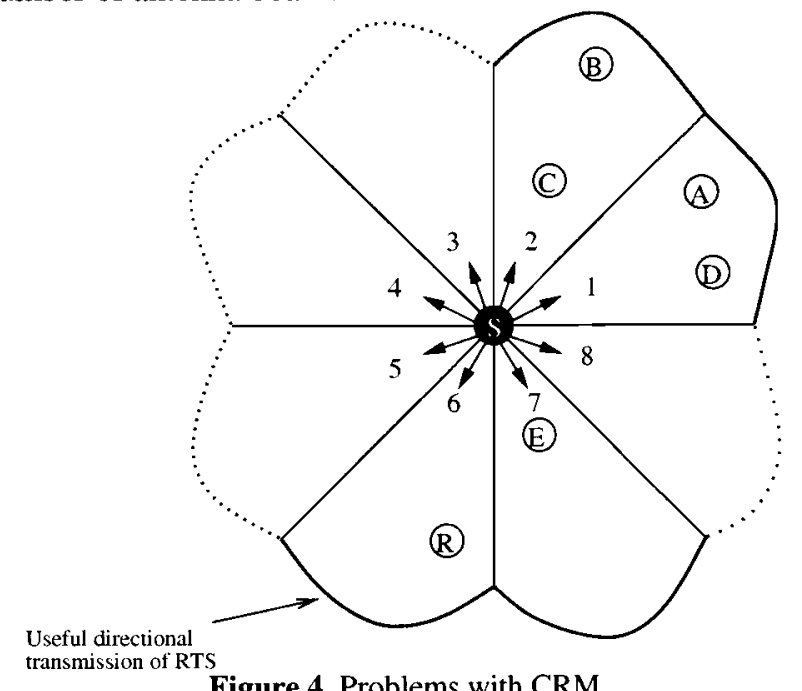

Figure 4. Problems with CRM

\section{THE PROPOSED DAMA PROTOCOL}

The DAMA protocol aims to effectively overcome the limitations found in both DMAC and CRM by utilizing a new combination of adaptive mechanisms. To take advantage of the increased gain obtained by directional antennas, all transmissions in DAMA are directional. Secondly, DAMA does not rely on prior availability of neighbors' location, while it learns its neighbors with time as communication between nodes takes place.

To prevent node deafness and the new types of hidden node problems aforementioned, DAMA employs circular directional transmission of both RTS and CTS. DAMA ransmits the RTS and CTS packets through the antenna beams with neighbors. In order to accomplish that, DAMA employs an adaptive mechanism where it learns and caches information about those sectors with neighbors. Initially, DAMA performs similar to CRM by sweeping through all antenna beams. However, as responses are received, it collects and caches neighboring information. To make the protocol simple in implementation, DAMA design has been inspired by the IEEE 802.11 MAC.

\subsection{DETERMINATION OF NEIGHBORS' LOCATION}

One important component in the design of DAMA is the precise determination of the location of a node. That is, DAMA carries out a continuous process of determining through which antenna a given neighbor can be reached. Here, DAMA relies on the very basic characteristics common to the majority of routing protocols employed over ad hoc networks $[21,22,24,24,25]$ : the use of broadcasting. These protocols either employ a form of periodic one-hop hello packets, or at least they flood the network with route request packets before data packets can be sent. The bottom line to note in these protocols is that, before any actual data communication can take place, a network layer broadcast must be carried out either by hello 
packets or by flooding routing requests control packets. From the MAC layer perspective, the routing broadcast packets are mapped onto MAC layer broadcasts to be transmitted to a node's neighbors.

Upon receipt of a network layer broadcast packet, a node, say $S$, initiates the circular directional transmission procedure of the broadcast packet through its, maybe all, antenna sectors. Assuming that the nodes possess a $M$-beam antenna array, the node $S$ has to first determine how many of these sectors are actually in idle state, that is, the DNAV (explained in detail later) in the direction $i, 1 \leq i \leq M$, is zero. The reason for this is that a packet cannot be sent in a busy sector or there will be a collision with any ongoing transmission is this particular busy sector. Let $K$ be the number of idle sectors, hence the sender node $S$ includes in the broadcast packet the value $(K-c-1)$ where $c$ is an integer (initially equal to zero) that keeps track of how many sectors the broadcast packet has been already sent. At the receiver side, the broadcast packet takes a time $T_{b c}$ to be completely received. The receiver, say node $R$, then waits for an additional time equals to $(K-c-1) * T_{b c}$ before initiating any transmission in the direction from which it received the packet from node $S$, say $D_{S}$. The rationale for carrying out such procedure is to give enough time for node $S$ to fully transmit all broadcast packets before any of its neighbor nodes can initiate a transmission in the direction $\mathrm{D}_{\mathrm{S}}$. In addition, the receiver node $R$ immediately caches the information that node $S$ can be reached through the antenna beam corresponding to the direction $\mathrm{D}_{S}$.

If the routing protocol employs any form of hello packets, all network nodes will eventually determine al] their neighbors during the learning phase, given that hello packets are periodically transmitted. More important than determining all particular neighbors of a node, this process also allows a node to determine if some of its sectors have any neighbors at all. On the other hand, if the protocol is not based on hello packets but uses flooding as a means to discover destination nodes, DAMA proceeds in a similar manner as described while the only difference resides on the way the route discovery procedure is carried out. According to the majority of on-demand routing protocols for ad hoc networks, once a node initiates a route discovery, all or most of its one-hop neighbors will eventually rebroadcast the route request packet. Therefore, the extra time $(K-c-1)^{*} T_{b c}$ waited by the receiving nodes before rebroadcasting the route request packet serves as a means to reduce collision, as well as to prepare the sender node $S$ to wait for the subsequent re-broadcast from its neighbors and consequently determine their locations. According to our simulations, we have noted that very few broadcasts are necessary for a node to figure out all of its neighbors. More specifically, up to three broadcasts are needed for a node to successfully figure out all of its neighbors.

\subsection{THE OPTIMIZED CIRCULAR DIRECTIONAL RTS AND CTS}

The DAMA protocol is based on a novel and optimized form of RTS and CTS transmission. Based on the neighbors' location described previously, RTS and CTS handshake can be optimized. One solution would be to send RTS and CTS only through the sector where the intended node is located as in DMAC, but this approach has its own limitations as previously described such as hidden node problems and deafness. Sweeping the entire antenna array through a circular directional transmission of RTS/CTS is also not a good solution, given that this approach will render the protocol increasingly inefficient as the number of antenna beams increase as observed in our simulations results to be presented later in this paper.

Therefore, in DAMA we optimize the RTS and CTS transmission by sending these control packets only through those sectors where nodes are found. This information is obtained through the neighbors' location procedure described in the previous subsection. Assuming that the number of antenna beams nodes have is equal to $M$ and that the direction $\mathrm{D}_{\mathrm{R}}$ node $S$ uses to communicate with node $R$ is currently idle, the sender node $S$ and receiver node $R$ will transmit $K_{S}$ and $K_{R}$ RTS and CTS packets, respectively, where $1 \leq K_{S} \leq M$ and $1 \leq K_{R} \leq M$.

Another important aspect in the design of DAMA is that the first RTS sent is always transmitted in the sector where its intended neighbor is located, and the circular directional RTS and CTS procedure is only initiated once the RTS/CTS handshake is successfully completed. We do this to overcome one of the limitations in CRM that initiates the circular directional transmission of the RTS packet (thus reserving the channel) before the sender node knows if any of its RTS has or will ever be correctly received by its intended destination node. Therefore in DAMA the sender node $S$ waits for the receiver node to send back its CTS before initiating its circular transmissions. In case CTS is not received, the sender times out and retransmits the RTS as in IEEE 802.11. This mechanism is illustrated by Figure 5 .

Upon reception of an RTS packet in step (1), the receiver proceeds similar to IEEE 802.11. That is, it waits for a period of time equal to SIFS and sends back a CTS as shown by step (2). Only after the RTS/CTS handshake is completed and the channel is reserved in their direction, will both sender and receiver nodes simultaneously initiate the circular directional transmission of their RTS and CTS packets, respectively, to inform their neighboring nodes. This simultaneous transmission of RTS and CTS is observed to save time and effectively takes care of the hidden node problem and deaf nodes at both the neighborhood of the sender and receiver. Figure 5 illustrates the simultaneous circular transmissions through step (3), where we note that nodes $S$ and $R$ do not send their RTS and CTS through all sectors, but only through those where neighbors can be found.

One issue still remains as to how sender and receiver again synchronize to carry out DATA transmission. To this end, the sender node $S$ includes in its RTS its value of $K$, that is, $K_{S}$, and the receiver node $R$ includes $K_{R}$ in its CTS back to node $S$. Through $K_{S}$, node $R$ is able to determine the exact point in time when node $S$ will have finished its circular directional transmission of RTS and hence will start transmitting DATA. Similarly, with $K_{R}$ node $S$ can precisely tell the moment node $R$ will be ready and waiting for DATA transmission. Clearly, this procedure works extremely well in uniformly distributed networks when $K_{S}$ is equal or approximately equal to $K_{R}$ or even when there is a very small discrepancy in their difference. When there is a 
large difference between $K_{S}$ and $K_{R}$, one node will eventually have to wait until the corresponding node is ready. Despite of this fact, this scheme is observed to perform well in most of the scenarios. Steps (4) and (5) in Figure 5 depict the DATA/ACK transmission.

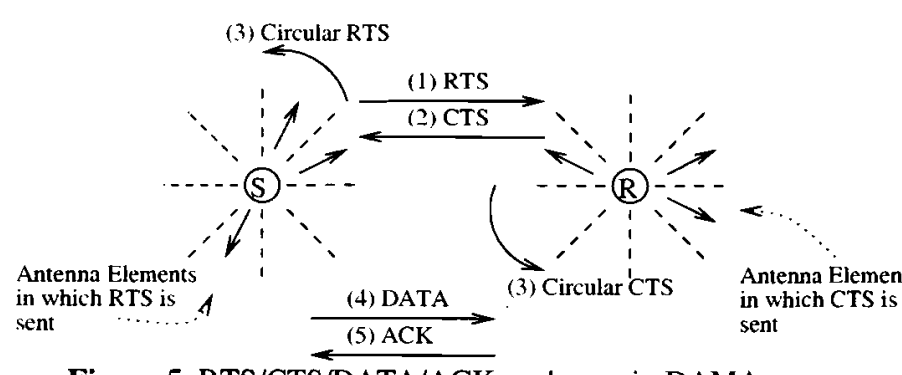

Figure 5. RTS/CTS/DATA/ACK exchange in DAMA

\subsection{THE DIRECTIONAL NAV (DNAV)}

As discussed in Section 4.1.1, one of the main problems with directional antennas is the new instances of the hidden terminal problem. To cope up with this problem, DAMA employs a combination of circular directional RTS and CTS (as explained earlier) together with a scheme that informs the neighbors of a node about its intended oncoming transmission. Upon receiving a circular directional RTS/CTS, a node has to decide if it is necessary to defer transmission in any direction so as to prevent collisions.

A discussion about this problem can be found in $[3,12]$ where a Directional NAV (DNAV) scheme [11] is employed to handle such issue. DNAV is an extension to the NAV concept used in IEEE 802.11 for directional antennas. Essentially, DNAV is a table that keeps track for each direction the time during which a node must not initiate a transmission through this direction. As in IEEE 802.11 , nodes continuously update this table upon overhearing a packet transmission in order to keep it from transmitting through this particular direction and generating collisions.

In order to ensure the correct update of DNAV, it does not suffice to only update it in the direction through which a packet has been received. When a node receives directional RTS/CTS packet, it should not only defer in the direction from which it received the packet so as to overcome the deafness problem, but also in the direction of the transmission between the sender and receiver. This problem is simplified in [3] as it is assumed knowledge of nodes' locations. The solution suggested in [12] is for a node to update its DNAV at antenna through which it received a RTS, CTS, or DATA packet. However, a node $T$ which receives a RTS may not necessarily receive the corresponding CTS, and vice-versa. The net effect of this is that node $T$ will update its DNAV in only one direction while it may still transmit, and eventually cause collisions, in the other directions. Therefore, this approach will only be valid when node $T$ is able to listen and decode both the RTS and CTS packets.

DAMA solves these problems by a very simple mechanism by which whenever a node receives a circular directional packet (i.e., RTS or CTS) it can reliably determine the antenna beams it should update its DNAV.
More specifically, whenever a node $S$ transmits an RTS or CTS packet to node $R$, it puts in the packet header the antenna beam node $R$ will use to receive node $S$ 's packet. Node $S$ can easily determine node $R$ 's receiving antenna, say $\theta_{R S}$, given that it knows its angle of arrival (AoA) [12], say $\theta_{S R}$, it uses to communicate with node $R$ by:

$$
\Theta_{R S}\left(\theta_{S R}\right)=\theta_{S R}+\Pi
$$

Assuming the nodes are using the same number of antenna beams, $\Theta_{R S}$ can be used by Node $S$ to determine node $R$ 's receiving antenna. For even number of antenna beams, equation (1) can be further simplified as a function of antenna beam. For example, Node $S$ can easily determine node $R$ 's receiving antenna, say $A_{R S}$, given that it knows through which antenna, say $\mathrm{A}_{S R}$, it uses to communicate with node $R$ by:

$$
A_{R S}\left(A_{S R}, M\right)=\left\{\begin{array}{l}
A_{S R}+\frac{M}{2}, \text { if } A_{S R}<\frac{M}{2} \\
A_{S R}-\frac{M}{2}, \text { otherwise }
\end{array}\right.
$$

where $M$ is the number of the antenna beams in a node, as previously defined. Basically, equation (2) is used to shift node S's antenna and obtain node $R$ 's receiving antenna. Now assume a neighbor of node $S$, say node $T$, receives the circular directional RTS packet through antenna beam $A_{T S}$. First of all, node $T$ updates its DNAV with the duration field contained in the RTS packet in the direction of node $S$, that is, it updates $\operatorname{DNAV}\left(A_{T S}\right)$. Next, node $T$ has to determine if it needs to update its DNAV for the same duration in the direction of node $R$ as well. For this to happen, node $R$ has to be a neighbor of node $T$ (explained in Section 5.1), and the antenna beam, say $A_{R T}$, node $R$ uses to communicate with node $T$ is equal to the antenna beam $A_{R S}$ contained in the RTS packet header. To calculate $A_{R T}$, node $T$ employs the same equation (2) and uses $A_{T R}$, the locally available antenna beam node $T$ uses to communicate with node $R$, as input.

To better illustrate this, consider the example in Figure 6. In this figure, node $S$ starts a transmission to node $R$ by first sending its RTS and receiving a CTS back from node $R$. In this figure, nodes $C$ and $E$ are neighbors of both node $S$ and $R$, node $D$ is a neighbor of node $S$ only, and nodes $A$ and $B$ are neighbors of node $R$ only. Besides the addresses of nodes $S$ and $R$, the circular directional RTS from node $S$ will contain the beam number three, as this is the value estimated by node $S$ through which node $R$ will receive node $S$ 's packet.

Upon receiving the RTS packet, nodes $C$ and $E$ first set their DNAV in the direction the RTS packet has been, (i.e., beam number four for node $E$ and beam number three for node $C$ ) received so as to prevent deafness, and then examine their neighbor information to determine if they can cause any collision with the reception in node $R$. The DNAV is set in the corresponding direction if a match is found.

In receiving the RTS packet, node $C$ realizes, through equation (1), that node $R$ is one of its neighbors and that it 
communicates with node $R$ through antenna number three, which happens to be equal to the antenna number contained in the RTS packet header sent by node $S$. Node $C$ then concludes that it can interfere in the reception at node $R$, thus updating its DNAV at antenna beam one (i.e., towards node $R$ ) for the corresponding duration field contained in the RTS packet header. A similar procedure is carried out at node $E$, which realizes that node $R$ can hear it through antenna beam two. Since the antennas are different, node $E$ realizes that it cannot harm nodes $S$ and $R$ transmission and hence does not update its DNAV towards node $R$. As for node $D$, when it receives the circular RTS from node $S$, it verifies that node $R$ is not one of its neighbors and, similar to node $E$, also does not update its DNAV.

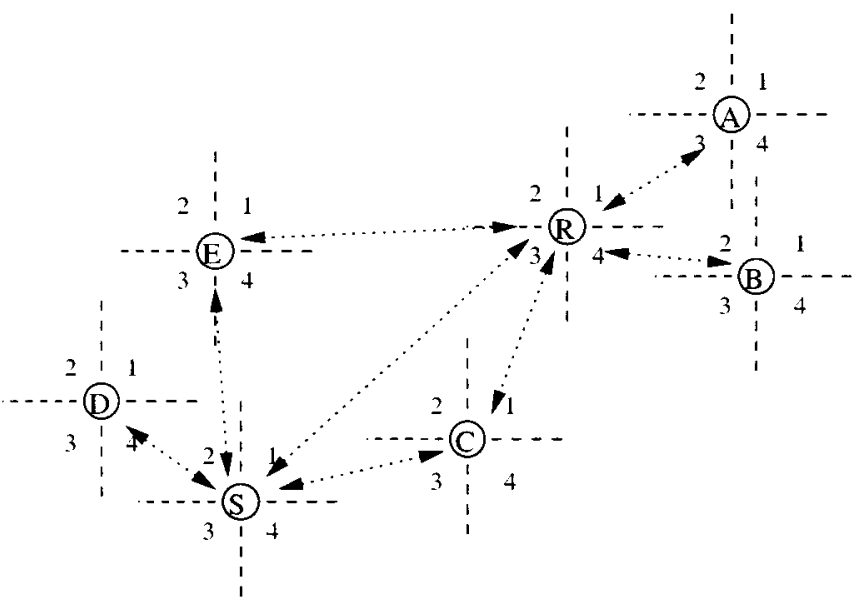

Figure 6. DNAV update procedure in DAMA

As for nodes $A$ and $B$, they cannot receive the circular directional RTS packet coming from node $S$, but will receive the circular directional CTS packet from node $R$. Upon receiving a CTS packet, nodes $A$ and $B$ carry out the same procedure as previously described, but now with respect to node $S$ and, if necessary, update their DNAV accordingly.

With this scheme, DAMA is able to effectively tackle the deafness at both sender and receiver neighborhood as well as minimize the possibility of collisions with the ongoing transmission. It is to be noted that in DAMA we assume that all the nodes in the network have the same number of antenna beams. In real life scenarios, however, the number of antenna beams supported by each node may be different. One way to extend DAMA to support such system is to include the number of beams being supported by a node at MAC layer packet transmissions. This way, a node is capable of not only keeping neighbor information, but also the number of beams used by each of its neighbors (equation (1) and (2)).

\subsection{IMPLEMENTATION DETAILS}

In this section we describe some important implementation details of DAMA. During the protocol implementation, we had to make adjustments to the timings and variables used in IEEE 802.11 given the new schemes of circular directional transmissions of RTS and CTS, and the use of $M$ directional antennas that requires that some of the default values of IEEE 802.11 variables be reconsidered. In the following subsections, we assume node $S$ is the transmitter and node $R$ is the receiver.

\subsubsection{SIMULATANEOUS TRANSMISSION OF RTS AND CTS}

As in IEEE 802.11, nodes in DAMA overhearing the RTS or CTS coming from nodes $S$ and $R$ update their DNAV based on the duration field contained in the packet. As multiple RTSs and CTSs may have to be transmitted, the duration field has to be incremented by these additional transmissions. Assume that $K_{S}$ and $K_{R}$ are the number of antenna beams through which the RTS and CTS packets have to be sent at nodes $S$ and $R$ respectively. The duration field has to be incremented by $\left(K_{S}\right.$ c) *RTS_Transmission_Time in the RTS packet, and by $\left(K_{R}-C\right){ }^{*}$ CTS_Transmission_Time in the CTS packet, where $c$ is an integer, initially equal to zero, maintained by each node that is incremented every time a circular directional packet is sent. This way, nodes overhearing the RTS or CTS can correctly set their DNAV for the entire duration of the transmission including the circular process.

\subsubsection{SHORT RETRY LIMIT (SRL)}

As explained in Section 4.1.1, the SRL is a threshold maintained by IEEE 802.11 that controls the number of packet (RTS or DATA) transmission attempts made before the send failure is reported to the routing layer. The way SRL has been set in IEEE 802.11 assumes an omnidirectional antenna is in place. However, when directional antennas are employed, the SRL can no longer be used to represent all antenna beams or an excess number of send failures may end up being reported. Therefore, we have extended the SRL to be one per each antenna beam, where the default value employed by NS IEEE 802.11 implementation (i.e., SRL $=7$ ) is now separately set for each antenna beam.

\subsubsection{CIRCULAR DIRECTIONAL TRANSMISSIONS AND DNAV}

As we have seen earlier, before the transmission of a RTS or CTS, nodes employing DAMA first analyze which of its sectors are both free and have any neighbors before calculating their value of the $K$ parameter. However, it might so happen that a sector becomes busy, if previously idle, or idle, if previously busy, after such an analysis has been performed. In the former case, a node stays silent for the corresponding transmission period if its DNAV became non-zero in a particular direction. For example if the DNAV of node $S$ or node $R$ is non-zero towards a specific direction, the RTS or CTS packet cannot be transmitted and these nodes stay silent for the corresponding transmission period. In other words, node $S$ stays silent for a period equivalent to RTS_Transmission_Time, and node $R$ stays silent for CTS_Transmission_Time. This way, collisions with other ongoing transmissions are prevented.

In the latter case, although very unlikely as we have observed through our simulation studies, a node skips a sector which was initially busy and became idle in the 
meantime. The reason for this is that in DAMA nodes $S$ and $R$ exchange their $K$ values so that this can be used for determination of their rendezvous point, and this commitment cannot be broken.

\section{PERFORMANCE EVALUATION}

We have implemented a directional antenna module in NS (version 2.26). This module models most of the aspects of a directional antenna system including variable number of antenna beams, different gains for different number of antenna beams among others. As for the protocol support, we have implemented DMAC, CRM, and DAMA.

For the simulations that follow, we have considered CBR traffic sources at data rates of $400 \mathrm{Kbps}, 800 \mathrm{Kbps}$, $1200 \mathrm{Kbps}$, and $1600 \mathrm{Kbps}$, and we measure the total network aggregate throughput of all flows. In addition, we evaluate DMAC, CRM and DAMA for six, twelve, and eighteen antenna beams with transmission ranges of 460 , 740 and 900 meters, respectively. For IEEE 802.11 , the transmission range is set to 250 meters. Also, in all the scenarios we consider a 2 Mbps network with no node mobility.

\subsection{LINEAR TOPOLOGY}

As discussed in Section 4.1.1, one of the problems in directional antennas is the hidden terminal problem due to asymmetry in gain. This problem is illustrated in Figure 7 , where node $S$ sends a RTS to node $R$, and has node $A$ as its neighbor. As we know, when nodes $S$ and $A$ are in idle mode, they hear omnidirectionally (i.e., with gain $\mathrm{G}^{\mathrm{O}}$ ). Since node $R$ 's beam includes node $S$ only (see Figure 7), node $A$ becomes a hidden node for $R$. If circular directional transmission of RTS is not employed (e.g., as in DMAC), node $A$ will not receive node $S$ 's RTS. Neither will node $A$ receive node $R$ 's CTS as it is listening the channel omnidirectionally. As a result, node $A$ will not be aware of the transmission between nodes $S$ and $R$. When node $S$ initiates the DATA transmission to node $R$, node $R$ uses selection diversity and starts to receive the RTS packet directionally with gain $\mathrm{G}^{\mathrm{d}}$. If during node $S$ 's DATA transmission to node $R$, node $A$ sends a packet towards node $R$ (e.g., a RTS to node $S$ ), node $R$ will receive the packet as it is in directional mode (i.e., with gain $G^{d}$ ), hence causing a collision with node $S$ 's DATA transmission. Note that although we have discussed this scenario with respect to node $A$, but the same problem occurs at node $B$ if circular directional transmission of CTS is not employed (e.g., as in both DMAC and CRM). DAMA deals with this problem effectively by employing an optimized circular directional transmission of both RTS and CTS, and hence informing nodes $A$ and $B$ about the intended transmission. Nodes $A$ and $B$ will, in turn, set their DNAVs in the direction of both nodes $S$ and $R$, thereby preventing collisions.

To quantitatively analyze the impact of this scenario on the network performance, we simulate the network of Figure 7 where we compare the performance of IEEE 802.11, DMAC, CRM, and DAMA. Since DMAC requires prior knowledge of neighbors' location, we have provided all protocols with such information for a fair analysis. Also, we have employed an array with six antenna beams for each node, but we note that any number of beams would produce similar results given that the nodes are aligned. In this scenario, node $S$ transmits to node $R$, node $A$ transmits to node $S$, and node $B$ transmits to node $R$. The coverage range is such that node $S$ 's RTS does not include node $B$, and node $R$ 's CTS does not include node $A$, since nodes $A$ and $B$ listen to the medium omnidirectionally. On the other hand, node $A$ 's RTS includes node $R$ as node $R$ is listens to node $S$ (DATA transmission) directionally, and may cause collisions. Similarly, node $B$ 's RTS includes node $S$ as node $S$ listens to node $R$ (CTS and $\mathrm{ACK}$ ) directionally, and may also result in collisions.

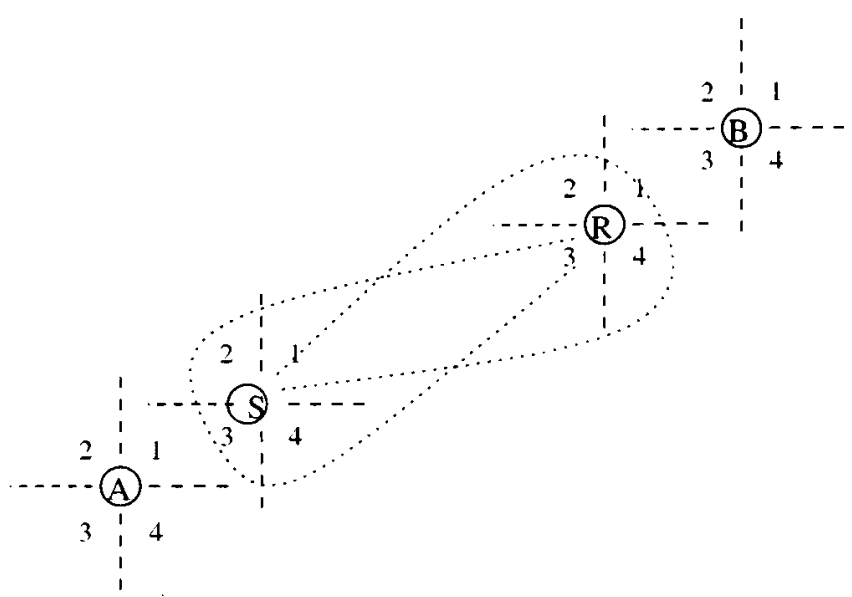

Figure 7. Example of a linear topology scenario

Figure 8 shows the simulation results obtained for this scenario. Similar to $[3,4]$, our results show that directional antennas have an inferior performance for linear topologies as compared to IEEE 802.11 given that the larger range is blocked in directional antennas as compared to IEEE 802.11. Thus, IEEE 802.11 achieves a better special reuse in linear topologies. Despite of that, we see that, amongst the directional MAC protocols evaluated, DAMA performs best. This is mainly due to the optimized circular directional transmission of both RTS and CTS which informs the neighbors of a node in little time about the intended transmission, thus preventing hidden terminals. CRM, on the other hand, does not perform comparable to DAMA as it employs circular transmission of RTS only, and does it in all sectors (even the "empty" ones). Finally, DMAC has the poorest performance as it causes many collisions due to the hidden terminals. 


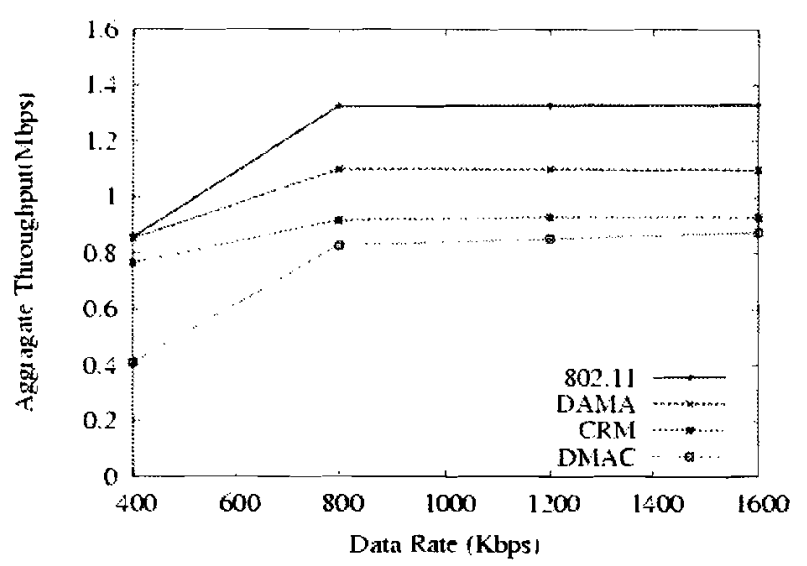

Figure 8. Throughput in a linear topology

\subsection{GAIN BY SPATIAL REUSE}

From now on, we concentrate on the performance comparison of IEEE 802.11, CRM and DAMA only, as these protocols do not assume prior knowledge of neighbors' location as in DMAC. Therefore, for the sake of a fair analysis and to compare the efficiency of CRM and DAMA neighbor discovery mechanisms, we have removed DMAC from the simulations that follow where no prior neighbors' location information is available.

In this section, we evaluate the performance of these protocols under scenarios where all nodes are within radio range of each other. Given that shortest transmission range is 250 meters in case of IEEE 802.11, the network topologies here evaluated have all nodes confined within a circle of 250 meters diameter. By doing this, we plan to evaluate the spatial reuse gain provided by directional antennas as compared to omnidirectional antennas. In the next section, we focus on the gain by increased coverage range.

\subsubsection{GRID TOPOLOGY}

The first topology we have used is a grid as shown in Figure 9. Here, a total of eight nodes and four flows are considered. Figure 9 depicts the various pairs of communicating nodes by connecting them with arrows, where the source of the arrow represents the transmitter and the sink the receiver.

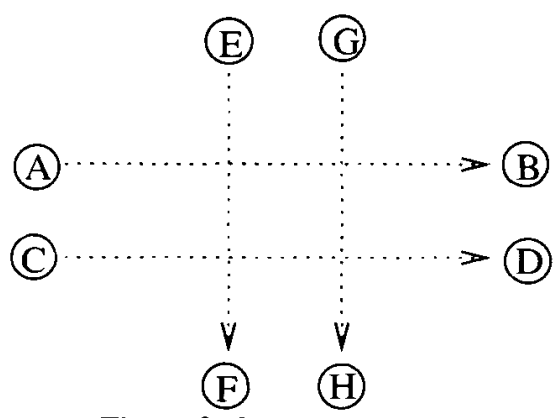

Figure 9. Grid topology

However, when the number of antennas is increased from six to twelve as depicted in Figure $10(\mathrm{~b})$, the possibility for spatial reuse increases as there is much less interference between the various transmission. As a consequence, DAMA boosts the network performance.
CRM, on the other hand, is shown to be inferior to IEEE 802.11 in low data rates, while becoming superior in medium to high loads. The reason for this is that CRM cannot benefit much from spatial reuse when the load is low as it spends a considerable amount of time performing the circular transmissions of RTS. When the load increases, however, CRM outperforms IEEE 802.11.

Finally, for eighteen antenna beams both DAMA and CRM are seen to outperform IEEE 802.11 as illustrated in Figure 10(c). Here, both protocols make full use of spatial reuse while DAMA achieves a much superior performance given its optimized circular RTS/CTS transmissions.

\subsubsection{RANDOM TOPOLOGY}

We now simulate a topology comprised of 16 nodes randomly distributed. Similarly to the previous study, here we consider all nodes are within radio range of each other. We have simulated a total of 10 scenarios and the results presented here are the average of their individual results.

Figures 11(a), 11(b), and 11(c) show the simulation results when nodes possess six, twelve, and eighteen antenna beams. It is important in these figures that IEEE 802.11 is practically the same when all stations are within the radio range of each other, as no spatial reuse is possible.

In Figure 11(a), we see that DAMA outperforms all other schemes, expect under low load as nodes in a random topology may eventually have to spend more time in the circular RTS/CTS procedure. In other words, in random topologies fewer are the "empty" sectors. However, in high load DAMA surpasses IEEE 802.11. It is interesting to note that CRM performance is inferior to IEEE 802.11. The reason is that it spends a lot more time than DAMA performing the circular transmissions. Not only this, it so happens that when a transmitter node using CRM is over performing all its circular transmissions, the RTS happened to have collided at its intended receiver. Thus, many circular RTS transmissions end up being useless.

When the number of antennas increases from six to twelve and eighteen (Figures 11(b) and 11(c)), we see that DAMA performance is further enhanced due to the increased spatial reuse. As for CRM, it surpasses IEEE 802.11 in medium and high load when twelve antennas are employed. However, in eighteen antenna beams CRM throughput is again below that of IEEE 802.11. Once more, the reason is that, as the number of antenna beams increase, CRM throughout is highly deteriorated given that it carries out far too many circular transmissions. In conclusion, we see that CRM is not a good solution when the number of antenna beams is high as it generates too much overhead.

\subsection{GAIN BY INCREASED COVERAGE RANGE}

Contrary to the previous section, here we focus on the second advantage of directional antennas, namely, the increased coverage range due to directionality. Therefore, in this section we evaluate the performance under scenarios where not all pairs of source and destination nodes are within radio range of each other. Given that IEEE 802.11 range is 250 meters, it may have to resort to the routing protocol in order to deliver a packet to a particular destination. On the other hand, it may be the case that CRM 


\section{A Directional Antenna Medium Access Control Protocol for Wireless Ad Hoc Networks Carlos de M. Cordeiro, Hrishikesh Gossain, and Dharma P. Agrawal}

and DAMA do not need to resort to routing as they can transmit for longer ranges. It is also possible for CRM and DAMA to use routing in case a direct link cannot be established, but the established route is likely to have fewer number hops as compared to IEEE 802.11. For the scenarios that follow, we have used the DSR routing protocol [25].

\subsubsection{GRID TOPOLOGY}

Here we study the performance in a grid topology similar to the one used in Section 6.1.1, but where the distance between pairs of source and destination nodes is selected between $[450,890]$ meters.

Figures 12(a), 12(b), and 12(c) present the aggregate throughput of all considered protocols in the case of six,

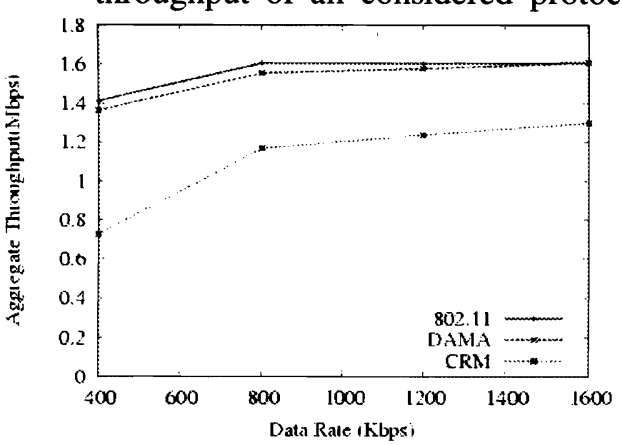

(a) -6 antenna beams

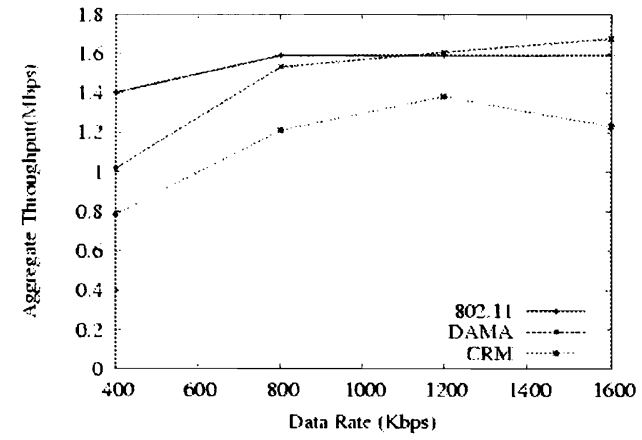

(a) -6 antenna beams

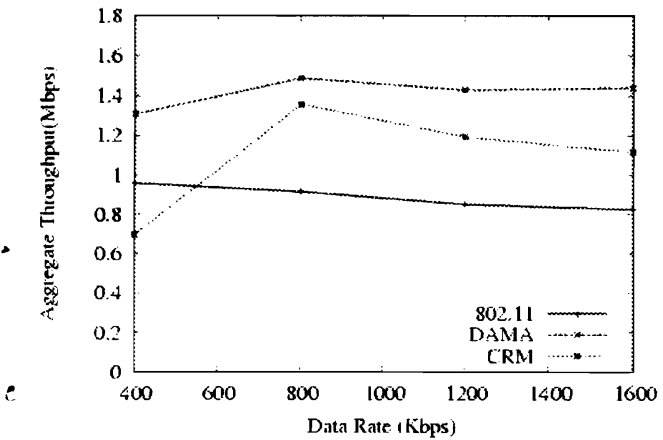

(a) -6 antenna beams

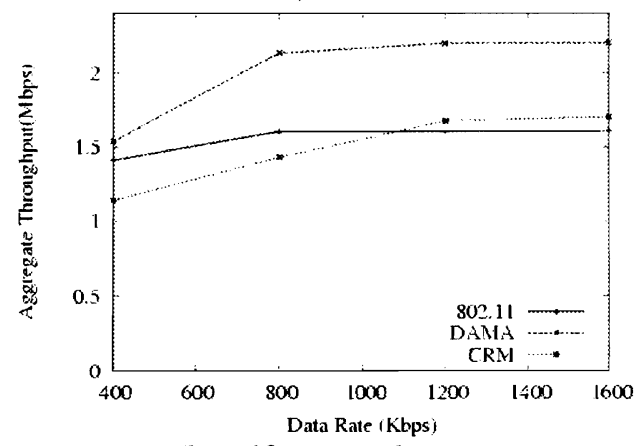

(b) - 12 antenna beams

Figure 10. Spatial reuse gain in grid topology

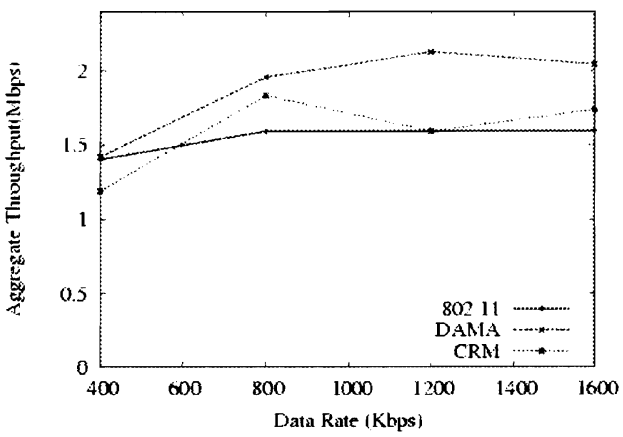

(b) - 12 antenna beams

Figure 11. Spatial reuse gain in random topology

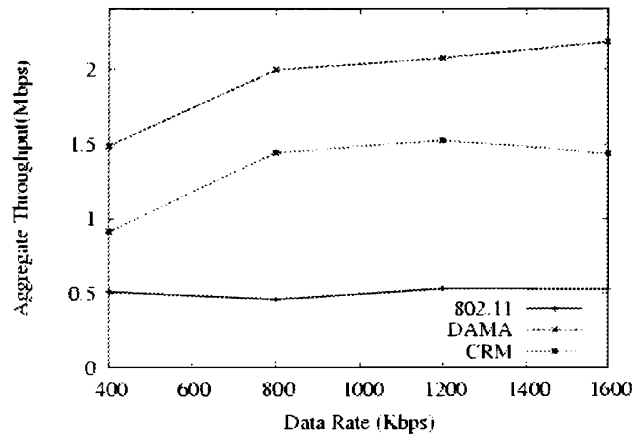

(b) - 12 antenna beams

Figure 12. Coverage range gain in grid topology

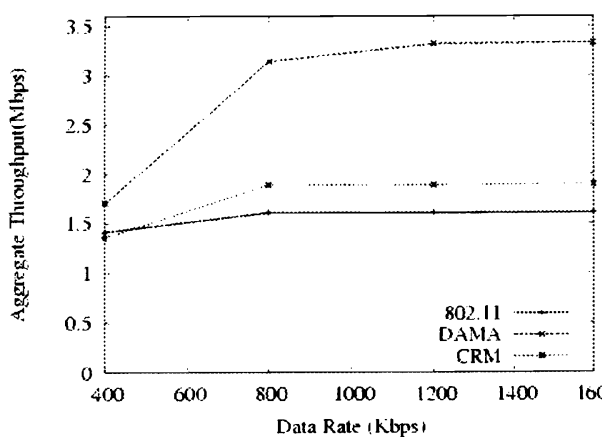

(c) - 18 antenna beams

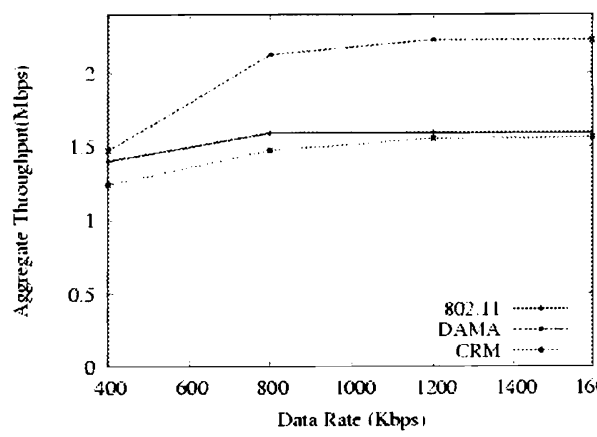

(c) - 18 antenna beams

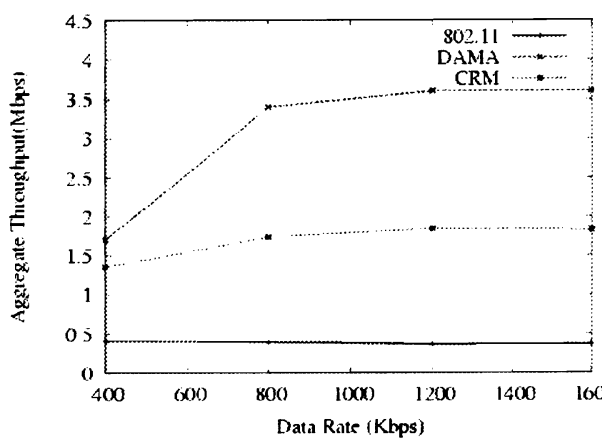

(c) - 18 antenna beams 


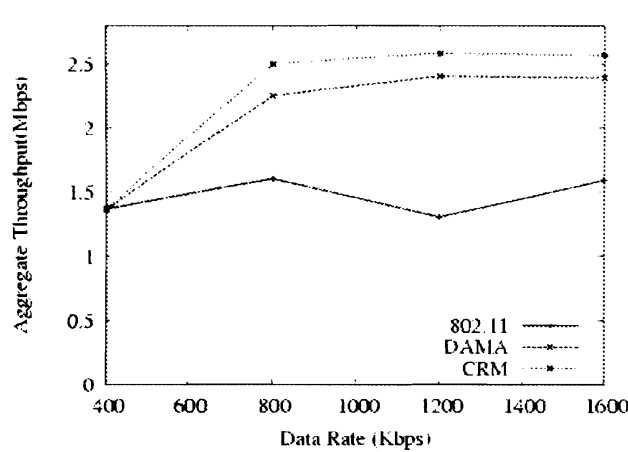

(a) -6 antenna beams

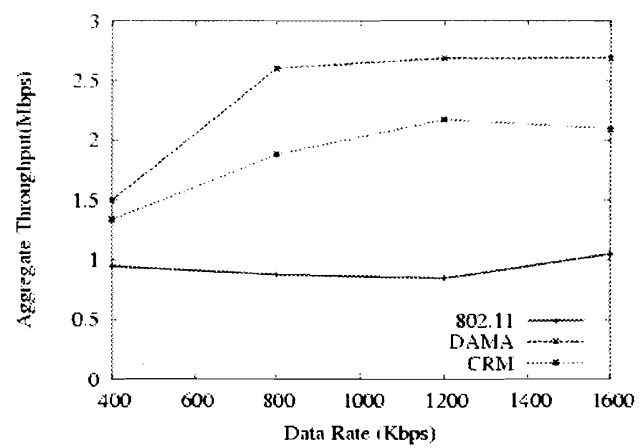

(b) - 12 antenna beams

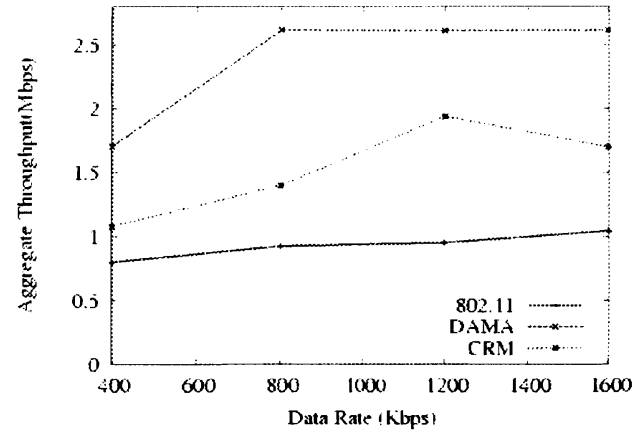

(c) - 18 antenna beams

Figure 13. Coverage range gain in random topology

\subsubsection{RANDOM TOPOLOGY}

Similar to the scenario used in Section 6.1.2, the scenario studied here reflects a total of 16 nodes randomly distributed on a two dimensional plane, where the distance between pairs of source and destination nodes is selected between $[450,890]$ meters. In addition, we have simulated a total of 10 scenarios and the results presented are the average of their individual results.

The results for six, twelve, and eighteen antenna beams are depicted in Figures 13(a), 13(b), and 13(c), respectively. As expected, in all three figures the directional protocols outperform the omnidirectional IEEE 802.11. For similar reasons to the previous section, here DAMA and CRM are able to find shorter routes between a pair of source and destination node. Notably, DAMA is shown to provide the best performance of all protocols considered.

\section{CONCLUSIONS AND FUTURE WORK}

In this paper we have considered the problem of medium access control for ad hoc networks employing directional antennas. We have discussed the shortcomings of existing work and have proposed a new protocol, called Directional Antenna Medium Access (DAMA), which implements unique mechanisms including simultaneous transmissions of RTS and CTS packets, an optimized form of sweeping. Through our extensive performance evaluation, we have observed that DAMA performs better than IEEE 802.11 and existing directional MAC protocols such as DMAC and CRM in all scenarios except in the linear topology. The linear topology case is particularly degrading to all directional MAC protocols, but DAMA is still observed to perform best in terms of all directional MAC protocols considered, while IEEE 802.11 performs best overall. Therefore, we see that the system performance depends upon the network topology as well as the traffic pattern between nodes.

As future work, we plan to investigate the issue of power control over directional antennas. Moreover, we are also looking into an integrated directional antenna aware routing and MAC layer design that could possibly take full advantage of the increased spatial reuse and coverage range provided by directional antennas systems.

\section{ACKNOWLEDGMENTS}

This work has been supported by the Ohio Board of Regents Doctoral Enhancement Funds and the National Science Foundation under grant CCR-113361.

\section{REFERENCES}

[1] IEEE Std. 802-11. "IEEE Standard for Wireless LAN Medium Access Control (MAC) and Physical Layer (PHY) Specification," June 1997.

[2] C. Fullmer and J.J. Garcia-Luna-Aceves, "Floor Acquisition Multiple Access (FAMA) for packet radio networks," Computer Communication Review, October 1995.

[3] R. Choudhury, X. Yang, R. Ramanathan, and N. Vaidya, "Using Directional Antennas for Medium Access Control in Ad Hoc Networks," in ACM Mobicom, September 2002.

[4] T. Korakis, G. Jakllari, L. Tassiulas, "A MAC protocol for full exploitation of Directional Antennas in Ad-hoc Wireless Networks," in ACM Mobihoc, June 2003.

[5] A. Chandra, V. Gummalla, and J. Limb, "Wireless Medium Access Control Protocols," IEEE Communications Surveys and Tutorials, vol.3, no. 2, 2000.

[6] M. Horneffer and D. Plassmann, "Directional Antennas in Mobile Broadband Systems," IEEE Infocom, April 1996.

[7] T. Yum and K. Hung, "Design Algorithms for Multihop Packet Radio Networks with Multiple Directional Antennas," IEEE Transactions on Communications, vol. 40, no. 11, 1992.

[8] A. Nasipuri, S. Ye, J. You, and R. Hiromoto, "A MAC Protocol for Mobile Ad Hoc Networks using Directional Antennas," in Proceedings of IEEE WCNC, September 2000.

[9] R. Choudhury, X. Yang, R. Ramanathan, and N. Vaidya, "Using Directional Antennas in Ad Hoc Networks," Final report submitted by Texas A\&M University to BBN technologies, July 2001.

[10] Y. Wang and J.J. Garcia-Luna-Aceves, "Spatial Reuse and Collision Avoidance in Ad Hoc Networks with Directional Antennas," in IEEE Globecom, November 2002. 
[11] Y.-B. Ko, V. Shankarkumar, and N. Vaidya, "Medium access control protocols using directional antennas in ad hoc networks," in IEEE Infocom, vol. 1(3), pp: 1321, Tel Aviv, Israel, 2000.

[12] M. Takai, J. Martin, A. Ren, R. Bagrodia "Directional Virtual Carrier Sensing for Directional Antennas in Mobile Ad Hoc Networks," in ACM MobiHoc, June 2002.

[13]R. Ramanathan, "On the performance of Ad Hoc Networks with Beamforming Antennas," in ACM MobiHoc, October 2001.

[14]M. Cooper and M. Goldburg "Intelligent Antennas: Spatial Division Multiple Access," Annual Review of Communications, pp: 999-1002, 1996.

[15]G. Foschini, M. Gans, "On limits of wireless communications in a fading environment when using multiple antennas," Wireless Personal Communication, 1998.

[16]C. Liberti and T. Rappaport, "Smart Antennas for Wireless Communications: IS-95 and Third Generation CDMA Applications," Prentice Hall, April 1999.

[17] NS-2 Network

Simulator, http://www.isi.edu/nsnam/ns/index.html.

[18]C. L. Fullmer, J. J. Garcia-Luna-Aceves, "Solutions to Hidden Terminal Problems in Wireless Networks," in ACM SIGCOMM, 1997.

[19] W. Moh, D. Yao, and K. Makki, "Wireless LAN: Study of hidden terminal effect and multimedia support", in Proc. Computer Communications and Networks, pp.422-431, 1998.

[20]E.-S. Jung and N. Vaidya, "A Power Control MAC Protocol for Ad Hoc Networks," in ACM Mobicom, 2002.

[21] C. Perkins, E. Royer, and S. Das, "Ad Hoc On Demand Distance Vector Routing (AODV)," Internet Draft, March 2001 (work in Progress).

[22] P. Jacquet, P. Muhlethaler, A. Qayyum, A. Laouiti, L. Viennot, T. Clausen, "Optimized Link State Routing Protocol," Draft-ietf-manet-olsr-04.txt.

[23] M. Gerla, G. Pei, X. Hong, T.-W. Chen, "Fisheye State Routing Protocol (FSR) for Ad Hoc Network," Draftietf-manet-fsr-01.txt, Nov. 2000.

[24] B. Bellur, R. Ogier, F. Templin, “Topology Broadcast Based on Reverse-Path Forwarding (TBRPF)," Draftietf-manet-tbrpf-01.txt, March 2001.

[25] D. Johnson, D. Maltz, Y.-C. Hu, and J. Jetcheva, "The dynamic source routing protocol for mobile ad hoc networks (DSR)," IETF Internet-Draft, Nov. 2001.

\section{BIBLIOGRAPHY}

CARLOS DE M. CORDEIRO is a Senior Research Engineer in Nokia Research Center where he is involved in the home networking arena and also with standardization activities within the IEEE 802.11 working group. He received his $\mathrm{PhD}$ in computer science and engineering in 2003 from the University of Cincinnati, $\mathrm{OH}$, USA, where he won the honorable Outstanding Doctoral Dissertation Award and the prestigious 2003/2004 The National Dean's List ${ }^{\circledR}$ Award. Earlier, he obtained a M.S. and B.Sc. in computer science in 1998 and 2000, respectively, from the Federal University of Pernambuco, Brazil. His research interests are in the broad area of wireless and mobile communication including MAC protocol analysis and design, MIMO systems, IEEE 802.11, IEEE 802.15, IEEE 802.16, cognitive radios, power control, spectrum management, ad hoc and sensor networks, routing, multicast, TCP over wireless, and cellular networks. Dr. Cordeiro has published numerous papers and holds pending patents involving MAC protocols for WLANs and WPANs. He has delivered tutorials in areas such as directional antenna systems, wireless broadband and mobile ad hoc and sensor networks, and in the past was the recipient of best paper awards from refereed networking conferences. Dr. Cordeiro has worked for IBM in San Jose, CA, and is a member of the IEEE.

HRISHIKESH GOSSAIN is a PhD candidate at the Department of ECECS, University of Cincinnati. He received B.E. in Electronics Engineering from Motilal Nehru Regional Engineering College, Allahabad India in 1998, where he was undergraduate Gold-Medallist of the College. He is co-inventor of 4 patents filled in QoS and e-media. His research interests include multicast, mobility management in wireless environment, Mobile IP, QoS, multimedia communications. He has previous work experience in Nortel Networks in Richardson, Dallas.

DHARMA P. AGRAWAL is the Ohio Board of Regents Distinguished Professor of Computer Science and Computer Engineering and the founding director for the OBR Research Center for Distributed and Mobile Computing in the Department of Electrical \& Computer Engineering and Computer Science, University of Cincinnati, $\mathrm{OH}$. He has been a faculty member at the Wayne State University, Detroit (1977-1982) and the N.C. State University, Raleigh, NC (1982-1998). His current research interests include wireless and mobile networks, distributed processing, and scheduling techniques. Dr. Agrawal is an editor for the Journal of Parallel and Distributed Systems and the International Journal of High Speed Computing. He has served as an editor of the IEEE Computer magazine, and the IEEE Transactions on Computers. He has been the Program Chair and General Chair for numerous international conferences and meetings. He was selected for the "Third Millennium Medal" by the IEEE for his outstanding contributions. Four of his patents in wireless networking area have also been approved recently. Dr. Agrawal is a fellow of the IEEE, ACM and AAAS. 